\title{
SURGICAL TACTICS IN RECURRENT INGUINAL HERNIAS AT THE CHILDREN
}

D0I: 10.36740/WLek202009222

\author{
Volodymyr M. Baibakov \\ DNIPRO MEDICAL INSTITUTE OF TRADITIONAL AND NON-TRADITIONAL MEDICINE, DNIPRO, UKRAINE
}

\begin{abstract}
The aim: Is to increase effectiveness of treatment at the children with recurrent inguinal hernias by improving surgical tactics.

Materials and methods: Method of laparoscopic correction of the recurrent inguinal hernias in children. For a period from 2017 to 2020 years in the surgical department of Municipal Establishment «Dnipro specialized clinical center of mother and child named by professor M. F. Rudnev» of Dnipro Regional Council» situated in the city Dnipro were carried out 48 laparoscopic procedures in a case of recurrent inguinal keel in children at the age of 3 years.

Results: We proposed an innovative method of laparoscopic correction the recurrent inguinal hernias, which allows to eliminate a hernial pocket without additional injury structures of the inguinal canal. At the analysis cases of recurrent inguinal hernias was concluded, that recrudescence appeared among young children and more frequently, after surgical interference on the strangulated inguinal hernias, which were operated by traditional ways.

Conclusions: According to the results of our research, it had been proved, that among numerous measures, focused on a restoration drainage system violation in the testicle, in a case of infringement obliteration of vaginal sprouts of a peritoneum in the boys and to keep safe ovarium vascularization in the girls.
\end{abstract}

KEY WORDS: inguinal hernia, recurrence, laparoscopy, children

Wiad Lek. 2020;73(9 p. II):2014-2016

\section{INTRODUCTION}

Relapses of inguinal hernias in children occuring at an early age, which is connected with difficulties of the anatomical and functional features in the structure of an inguinal region and immaturity of the connective tissues [1]. These factors determine technical difficulty of performing surgical intervention and demand from the surgeon a good knowledge of anatomy in the inguinal region at the children in the early age and high practical training $[2,3]$. In the analysis of the national and foreign literature sources $[4,5]$ from the given subject draws attention a lack of common pathogenic view, which focused not only in the separate issues, but on the following fundamental issues as:

- determination of the optimal terms of re-operation;

- choice of the most minimally invasive and optimal methods of surgical correction;

- optimization principles of the postoperative management of patients $[6,7,8]$.

When analyzing literature review and based on our own experience, we would come to the conclusion, that results of treatment recurrent inguinal hernias in children were not satisfy scientists and clinicians. It should be explained by a high percentage of postoperative complications, especially on the reproductive organs $[9,10,11]$. High traumatism of repeat surgical intervention in a groin area with scarring and disruption normal anatomic relationship between structures in a groin area should lead to the negative consequences on the reproductive organs at the boys and girls in a fertile age.
Therefore, using of laparoscopic correction of the recurrent inguinal hernia after traditional intervention in a groin area significantly reduces risk of additional trauma in the structures of an inguinal region in children. Solution of this problem is an important step in a direction of safety reproductive health of the nation and preservation of complete family $[12,13,14]$.

\section{THE AIM}

Aim of research is to increase effectiveness of treatment at the children with recurrent inguinal hernias by improving surgical tactics.

\section{MATERIALS AND METHODS}

Method of laparoscopic correction of the recurrent inguinal hernias in children. For a period from 2017 to 2020 years in the surgical department of Municipal Establishment «Dnipro specialized clinical center of mother and child named by professor M. F. Rudnev» of Dnipro Regional Council» situated in the city Dnipro were carried out 48 laparoscopic procedures in a case of recurrent inguinal keel at the children, which were undergoing the first intervention by traditional access in a groin area. All children were at the age of 3 years. Analysis cases of recurrent inguinal hernias had been shown that recrudescences appeared at the young children and more frequently, after surgical interference on the strangulated inguinal hernias, which were operated by traditional ways (95\%). 


\section{RESULTS}

The method is performed as follows. Under the endotracheal narcosis by transumbilical way we submit port D- $5 \mathrm{~mm}$, through which we conduct laparoscope D-5 mm into the abdominal cavity. Pressure of $\mathrm{CO}_{2}$ in the abdominal cavity is supported on the level 8-10 mm of mercury column. Examine the internal inguinal rings, we should established a presence of recurrent inguinal hernia. During applying of carboxiperitoneum, intraoperatively, we performed the ultrasound-control of a groin area from the side of surgical intervention. Evaluate presence of gas in a hernial pocket, its shape, size and location, condition of the aponeurosis. In a case where the hernial pocket is filled with gas in the stretch, and condition of aponeurosis corresponded to the plastic, we performed only suturing of an internal inguinal ring after hydraulic dissection and injection of tissue adhesive into the lumen of a hernia pocket. Performed hydraulic preparation of tissue around the internal inguinal ring with $0.9 \%$ sodium chloride solution. After that we proceed to the imposition of a purse-string suture in a place of internal inguinal ring. For this purpose we should used prolene $2-0$. We take in a seam peritoneum in the place, where were held hydraulic preparation of the tissues. Under a video control, in the point of projection an internal inguinal ring, this is determined by pressing on the anterior abdominal wall, we input to a groin channel the dulled end needle G-19 with prolene 2-0 thread, which is not absorbed. Hereafter we should apply hydraulic preparation with $0.9 \%$ sodium chloride solution carried out by the following way. Thread should pass under the peritoneum on one of the walls in the inguinal canal. Having enter a thread, we should form the loop. Then the needle is removed, and a loop is left in the abdominal cavity. The needle is inserted in the other end of the thread and through the same puncture thread is entered under the peritoneum opposite a wall of inguinal canal. The end of a thread should be passed through already formed loop.

Then remove a needle, both ends of a thread output on the anterior abdominal wall. Before tightening of a formed around the internal inguinal ring suture, to a lumen of the inguinal canal we injected one-component adhesive tissue with active substance of n-butylcyanoacrylate, after the node could be tighten.

The ends of a thread should be cut, estimate control of the purse-string suture tightness. Defect of aponeurosis eliminated by mini access in a groin area. For diagnosis of necrotic changes or if it is suspected, we recommend to continue operation by an open access. If, according to the ultrasound, there is divergence of aponeurosis with the next formation of defect, it should be carry out a mini skin incision to $2.0 \mathrm{~cm}$ in length and having sutured defect of aponeurosis without removal of a hernia pocket. Suturing of the internal inguinal ring was done after hydraulic dissection and injection of a tissue adhesive into the lumen of hernia pocket from the side of abdominal cavity.

Hydraulic preparation of tissues in a region of internal inguinal ring from the side of abdominal cavity with applying $0.9 \%$ $\mathrm{NaCl}$ solution should be used only as the additional method of prevention bleeding, as the result of vessels injury. Hydraulic preparation of the internal inguinal ring tissues, with using $0.9 \%$ $\mathrm{NaCl}$ solution, significantly reduces risk of the spermatic cord vessels injury in the in boys, and should prevent oncogenes of hydrocele or testicular atrophy.
$\mathrm{N}$-butylcyanoacrylate (enbucrilate) is medical glue in a liquid form, which facilitates drawing it through the lumen of hernia pocket with applying the biocompatible, haemostatic, antibacterial, non-histotoxic drug. It should prevent formation of secondary inflammatory responses, and blue color induces in the field of operational space. Its introduction into the lumen of hernia pocket increased reliability of the purse-string suture; eliminates risk of recurrence hernia, hydrocele or testicular atrophy in the boys, decreases time of surgical treatment. Properties of glue are sufficient to overcome efficiency, reduce morbidity and providing of a satisfactory cosmetic effect.

The average duration in a hospital was 2 days. High efficiency of the given method was confirmed clinically and with ultrasound control.

\section{DISCUSSION}

Results of research provide an opportunity to minimize in practice development of complications and decreased risk of postoperative complications. Thus, proposed method of laparoscopic correction of recurrent inguinal hernias allows to eliminate a hernial pocket without the additional injury structures of the inguinal canal, which are being in a condition of cicatricial process, forming after the previous surgery in the inguinal region. Besides, a good cosmetic effect of operation prevents re-formation of the adhesions and rough scars. Finally, advanced diagnostics and choice optimal method of surgical treatment the recurrent inguinal hernias in children should be actual issues. Scientific developments of recent years should be focused on feasibility of the anatomically and pathogenetically reasonable surgical interventions.

Review methods for the diagnosis of recurrent inguinal hernias in children was carried out. The diagnosis of recurrent inguinal hernias received a lot of attention in the world and national literature. There were used a variety of research methods. Pelviography has been proposed for the detection of recurrent inguinal hernias in children. With the development of science, new methods of examining patients appeared. Immediately after its appearance, ultrasonography occupied a firm place among the methods of recurrent inguinal hernias in children diagnostics, since it allowed determining the location, size and structure of the gonads with a sufficiently high accuracy. The use of the technique based on the doppler effect, which allows to investigate a blood flow in the testicular parenchyma. At the same time, the assessment is not only qualitative, but also quantitative, since it became possible to measure the blood flow parameters: blood flow velocity during systole, blood flow velocity during diastole, resistance index.

If ultrasound diagnostics of internal organs devoted many works described in the world literature, then the works that are devoted to the dopplerography of the gonads, especially in children, are rare. Meanwhile, this diagnostic method allows evaluating hemodynamic in the gonad at a qualitative and quantitative level, and indirectly judging the state of the testicular parenchyma. Diagnostic laparoscopy has become a method that has become an integral and indispensable part of the diagnosis of recurrent inguinal hernias in children and 
determination of treatment tactics. Minimally invasiveness in combination with highly informative made this method the leading one in diagnosing these forms of pathology.

Relapses of inguinal hernias we recommended to operate by laparoscopic way of surgery with an intraoperative ultrasound control. The main reasons of recurrence inguinal hernias in children we consider infringement of technique during the first operation. It should be the following disadvantages:

- removal only a distal part of the hernia;

- removal not a hernia pocket, but the surrounding tissues; - violation methods of suturing aponeurosis of the external oblique muscle in the abdomen;

- performing of surgical operations should be providing by pediatric surgeons, but not by the surgeons of general practice without sufficient experience.

Given factors give an opportunity for the pediatric surgeons to search new, less traumatic and highly effective methods of surgical treatment the recurrent inguinal hernias in children. These objectives correlated with proposed by us innovative method of laparoscopic correction the recurrent inguinal hernia with carrying out an intraoperative ultrasonography for an inguinal control of a groin area from the side of intervention.

\section{CONCLUSIONS}

1. In the recurrence of inguinal hernia numerous disorders of testicular blood flow at the boys and impaired ovarium vascularization at the girls are risk factors of infertility in the reproductive period.

2. Among measures, which focused on the restoration violations of testicle drainage system in a case of infringement obliteration of vaginal sprouts of a peritoneum in the boys and preserve ovarium vascularization in the girls during re-intervention a basic role takes implementation of the pathogenetically substantiated method of surgical correction pathology on the level of a deep inguinal ring with using modern laparoscopic techniques.

3. Ultrasound method of research with using of doppler technology is a highly efficient way of assessing the anatomical indicators as well as estimation size of testicles in boys and ovaries in girls, vessels, parenchyma of the reproductive organs, and functional indicators.

\section{REFERENCES}

1. Akbashev R. N. Laparoscopic treatment of strangulated inguinal hernias in children: author. dissertation on scientific degree PhD of medicine: 14.00.35 "Children's surgery". 2006; 23.

2. Gorbatyuk A.M. Materials of conference 30th anniversary of vascular surgery clinic in Lviv "Actual problems of angiology". 2000; 74.

3. Gorbatyuk A.M. General regularities of pathogenesis of male infertility, caused by disorders of vaginal sprouts of a peritoneum. Urology. 2000; 2: 47-49.

4. Carneiro P.M., Rwanyuma L. Occurrence of contralateral inguinal hernia in children following unilateral inguinal herniotomyt. East. Afr. Med. J. 2004; 81 (11): 574-576.

5. Schier F. Direct inguinal hernias in children: laparoscopic aspects. Pediatr. Surg. Int. 2010; 16 (8): 562-564.
6. Achtemichuk Y.T., Skoreyko P.M. Topographic-anatomic substantiation of surgical operations. Ukrainian morphological almanac. 2005; 3 (3): 3-8.

7. Stelmahovich V.N. Materials of Russian Symposium of Pediatric Surgeons "Surgery pathology of sexual development in children". 2007; 48.

8. Tan H.L. Laparoscopic repair of inguinal hernias in children. J. Pediatr. Surg. 2001; 36 (5): 880-833.

9. Grytsuliak B.V., Grytsuliak V.B., Khallo 0.E. State of macro - and microvasculature and parenchyma of the testis in terms of direct inguinal hernias at the men of reproductive age. Galician medical journal. 2010; $1: 26-27$

10. Dronov A.F., Poddubny A.F., Kotlobovskyi V.I. Endoscopic surgery in children. M.: GEOTAR-Med.; 2002: 437.

11. Bozhedomov V.A., Nikolaeva M.A., Ushakova I.V. Pathogenesis of decreased fertility in autoimmune reactions against spermatozoons. Obstetrics and gynecology. 2012; 8 (2): 64-69.

12. Grytsuliak B.V., Grytsuliak V.B., Gotur A.I. Structural and functional characteristics of the bloodstream and parenchyma of the testis in men aged 75-90 years. Scientific Bulletin of Uzhgorod National University. 2013; 1(46): 12-15.

13. Yatskiv A.G., Tarnovskaya A.T. Causes and forms of male infertility and diagnostic methods of the ejaculate as a main indicator of men's health. Bulletin of Lviv University. 2012; 60: 4-20.

14. Owings E.P., Georgeson K.E. A new technique for laparoscopic exploration to find contralateral patent processus vaginalis. Surg. Endosc. 2000; 14 (2): 114-116.

Research work was carried out within a framework the cathedral theme of the research work: "Morphological state of vascular-tissue relations in the structure of organs and tissues and development of organ-preserving operations using laser technique", state registration number 0104U010388 (implementation period 2017-2021 years).

\section{ORCID and contributionship:}

Volodymyr M. Baibakov: 0000-0001-8632-103X A, B, C, D, E, F

\section{Conflict of interest:}

The Author declare no conflict of interest.

\section{CORRESPONDING AUTHOR Volodymyr M. Baibakov \\ Dnipro Medical Institute of Traditional \\ and Non-Traditional Medicine \\ 13 Zadunaiska St, village Sursko-Lytovske, \\ 52064 Dnipro, Ukraine \\ tel: +380509198547 \\ e-mail:baybakov837@gmail.com}

Received: 03.10.2019

Accepted: 29.06 .2020

A - Work concept and design, B - Data collection and analysis, C - Responsibility for statistical analysis,

D-Writing the article, $\mathbf{E}$-Critical review, $\mathbf{F}$ - Final approval of the article 